ARTICLE

\title{
Group A PP2Cs evolved in land plants as key regulators of intrinsic desiccation tolerance
}

\author{
Kenji Komatsu ${ }^{1, \star, \dagger}$, Norihiro Suzuki ${ }^{1, \star}$, Mayuri Kuwamura', Yuri Nishikawa', Mao Nakatani ${ }^{1}$, \\ Hitomi Ohtawa', Daisuke Takezawa², Motoaki Seki ${ }^{3}$, Maho Tanaka³ ${ }^{3}$ Teruaki Taji ${ }^{1}$, Takahisa Hayashi ${ }^{1}$ \\ \& Yoichi Sakata ${ }^{1}$
}

\begin{abstract}
Vegetative desiccation tolerance is common in bryophytes, although this character has been lost in most vascular plants. The moss Physcomitrella patens survives complete desiccation if treated with abscisic acid (ABA). Group A protein phosphatases type $2 \mathrm{C}$ (PP2C) are negative regulators of abscisic acid signalling. Here we show that the elimination of Group A PP2C is sufficient to ensure $P$. patens survival to full desiccation, without ABA treatment, although its growth is severely hindered. Microarray analysis shows that the Group A PP2C-regulated genes exclusively overlap with genes exhibiting a high level of ABA induction. Group A PP2C disruption weakly affects ABA-activated kinase activity, indicating Group A PP2C action downstream of these kinases in the moss. We propose that Group A PP2C emerged in land plants to repress desiccation tolerance mechanisms, possibly facilitating plants propagation on land, whereas ABA releases the intrinsic desiccation tolerance from Group A PP2C regulation.
\end{abstract}

\footnotetext{
${ }^{1}$ Department of Bioscience, Tokyo University of Agriculture, 1-1-1 Sakuragaoka, Setagaya-ku, Tokyo 156-8502, Japan. ${ }^{2}$ Graduate School of Science and Engineering and Institute for Environmental Science and Technology, Saitama University, Saitama 338-8570, Japan. ${ }^{3}$ RIKEN Center for Sustainable Resource Science, 1-7-22 Suehiro-cho, Tsurumi-ku, Yokohama 230-0045, Japan. * These authors contributed equally to this work. $†$ Present address: Department of Bioproduction Technology, Junior College of Tokyo University of Agriculture, 1-1-1 Sakuragaoka, Setagaya-ku, Tokyo 156-8502, Japan. Correspondence and requests for materials should be addressed to Y.S. (email: sakata@nodai.ac.jp).
} 
A bscisic acid (ABA) has a major role in responses to waterrestricted conditions in flowering plants, including stomatal closure, adaptation of vegetative tissues to drought, high salinity and low temperatures, and acquisition of desiccation tolerance and subsequent dormancy of seeds ${ }^{1,2}$. ABA has been also suggested to have a role in vegetative desiccation tolerance, a common feature of bryophytes (the basal land plants) but evolutionarily lost in vascular plants ${ }^{3-5}$. Protonemata of the model moss Physcomitrella patens are tolerant only to moderate water stress-that is, water loss up to around $90 \%$ of its fresh weight, or about $-13 \mathrm{MPa}$ by water potentials ${ }^{6}$-but not to complete desiccation in experimental conditions ${ }^{6,7}$. However, ABA pretreatment markedly increases their tolerance to complete desiccation ${ }^{7}$ or equilibration to $13 \%$ relative humidity $(-273 \mathrm{MPa})^{6}$. The inductive desiccation tolerance in vegetative tissues of the basal land plants, such as $P$. patens, suggests the hypothesis that acquisition of sensing and response mechanisms for $\mathrm{ABA}$ by their ancestors was critical for invasion of and adaptation to land, where water potentials were fluctuating continuously. Recent key studies in the vascular plant Arabidopsis provide evidence of a pivotal role of Group A protein phosphatases type $2 \mathrm{C}(\mathrm{PP} 2 \mathrm{Cs})^{8-12}$ in the $\mathrm{ABA}$ signalling pathway ${ }^{13}$. In the absence of ABA, Group A PP2Cs act through the physical interaction with subclass III of plant-specific SNF1related protein kinases (SnRK2) and dephosphorylate the kinase activation loop, inhibiting ABA signalling transduction ${ }^{14-19}$. ABA binding to the receptor pyrabactin resistance 1-like (PYL) (also known as PYR or regulatory component of ABA receptor $)^{20,21}$ promotes the interaction between the receptor and the catalytic site of Group A PP2Cs ${ }^{22,23}$, leading to the inhibition of Group A PP2Cs and facilitating SnRK2 activation. It is evident that Group A PP2C is a central component in the induction of ABA signalling. Although genes for Group A PP2C are present only in the land plant lineage, they have expanded in number through land plant evolution. Group A PP2C of the flowering plant Arabidopsis consists of nine genes, whereas only two genes encode Group A PP2C in the moss $P$. patens. Therefore, the role of these two moss Group A PP2C genes could reflect ancestral functions. In fact, and as reported here, those two genes appear to be directly involved in $\mathrm{ABA}$ responses, including induced vegetative desiccation tolerance.

Here we establish a null plant for Group A PP2C for the first time, taking advantage of efficient gene targeting in $P$. patens. Analysis of the null plant reveals that Group A PP2C represses the intrinsic feature of tolerance to desiccation, freezing and salinity, and promotes proliferation of the vegetative tissue. We further demonstrate that Group A PP2C exclusively regulates genes that exhibit a high level of $\mathrm{ABA}$ induction. In addition, our results show that Group A PP2C acts downstream of $\mathrm{ABA}$-activated kinases in the moss. On the basis of these collective findings, we propose that Group A PP2C-mediated ABA signalling evolved in ancestral land plants to control preexisting mechanisms for desiccation tolerance, facilitating rapid propagation on land.

\section{Results}

Role of Group A PP2C in growth and development. We previously reported a loss-of-function analysis of $P p A B I 1 A$ single disruptants (ppabila) generated by gene targeting ${ }^{24}$. Either the wild-type (WT) or the ppabila plants were transformed with the $P p A B I 1 B$-targeting construct to generate $P p A B I 1 B$ single disruptants (ppabi1b) or PpABI1 double disruptants (ppabila/b), respectively (Supplementary Figs S1 and S2).

We first analysed the effect of null expression of Group A PP2C on growth and development of $P$. patens under normal conditions. Previously, we reported that the ppabila single disruptant showed normal growth and development in the absence of exogenous ABA. As expected, the ppabilb single disruptant also exhibited normal protonemal growth, as did WT and the ppabila single disruptant. However, the ppabila/b double disruptant showed a distinct phenotype in protonemal growth (Fig. 1a-d). The ppabila/b plants grew very slowly in a patchy fashion compared with the WT or both the single disruptants. Microscopic analysis of these filaments (Fig. 1e,i) revealed that each protonemal cell of the ppabila/b plants did not elongate like WT but differentiated into spherical brood cells, which are formed in the WT, only with exposure to exogenous ABA or to stress conditions ${ }^{25}$. Exogenous ABA has been known to prevent cytokinin-induced formation of buds that eventually develop into gametophores ${ }^{26}$. The protonemata of the ppabila/b, however, developed dwarf gametophores (Fig. 1f,j) at a frequency similar to that of WT. The gametophores of ppabila/b plants developed sporophytes indistinguishable from those of WT (Fig. 1g,k), and these spores also germinated (Fig. 1h,l). These results suggest that Group A PP2C is essential for proper growth, mainly for elongation of both protonemata and gametophores under non-stress conditions; however, it is not involved in developmental regulation, such as bud induction, gametophore development, embryogenesis or spore germination.

Role of Group A PP2C in stress responses. To test the role of Group A PP2C in vegetative desiccation tolerance, protonemata were desiccated rapidly by exposure to an atmosphere of about $-100 \mathrm{MPa}$ (about $50 \%$ relative humidity) in a laminar flow cabinet for $24 \mathrm{~h}$. This treatment resulted in almost complete removal of water (about $99 \%$ on a fresh weight basis) from the tissues of WT (as low as $0.056 \pm 0.002 \mathrm{~g} \mathrm{~g} \mathrm{dm}^{-1}$; dm, dry matter) and ppabila/b plants $\left(0.052 \pm 0.001 \mathrm{gg} \mathrm{dm}^{-1}\right)$. It has been reported that only ABA-treated WT plants can survive this extremely dry condition ${ }^{6}$. According to these previous results, WT or the both single disruptants could not be recovered from desiccation stress after the treatment; here, however, ppabila/b plants survived and regrew without the assistance of exogenous ABA (Fig. 2a,b). The findings demonstrated that Group A PP2Cs repress the intrinsic desiccation tolerance of $P$. patens.

We also compared the freezing tolerance of the doubledisruptant plants with that of WT or both single disruptants. Protonemal tissues of WT, both single disruptants, or double disruptants were exposed to subzero temperatures from $-5^{\circ} \mathrm{C}$ to $-15^{\circ} \mathrm{C}$ without $\mathrm{ABA}$ pretreatment, and the survival was calculated by measuring the electrolyte leakage (Fig. 2c). Protonemal cells of WT were not tolerant to freezing even at $-5^{\circ} \mathrm{C}$. A slight but significant increase of freezing tolerance of the ppabila plants but not the ppabilb plants was observed at $-5^{\circ} \mathrm{C}$. On the other hand, the ppabila/b plants showed about $90 \%$ survival at $-5^{\circ} \mathrm{C}$. The ppabila/b plants still showed about $40 \%$ survival even after freezing at $-15^{\circ} \mathrm{C}$. At this temperature, WT protonemata treated even with $100 \mu \mathrm{M}$ ABA for $24 \mathrm{~h}$ showed less than $20 \%$ survival (Fig. 2d).

We further tested the tolerance of the ppabila/b plants to salinity. Protonemal colonies of WT or transgenic plants were challenged with a high concentration of $\mathrm{NaCl}$ for 5 days, then recovered on normal media for 7 days (Supplementary Fig. S3). Growth of the ppabila/b plants was little affected by up to $450 \mathrm{mM} \mathrm{NaCl}$. Taken together, these results suggest that Group A PP2C alleviates constitutive tolerances of protonemata to waterassociated stresses in the absence of ABA.

Late embryogenesis abundant (LEA) proteins are hydrophilic proteins that specifically accumulate in developing seeds or the 


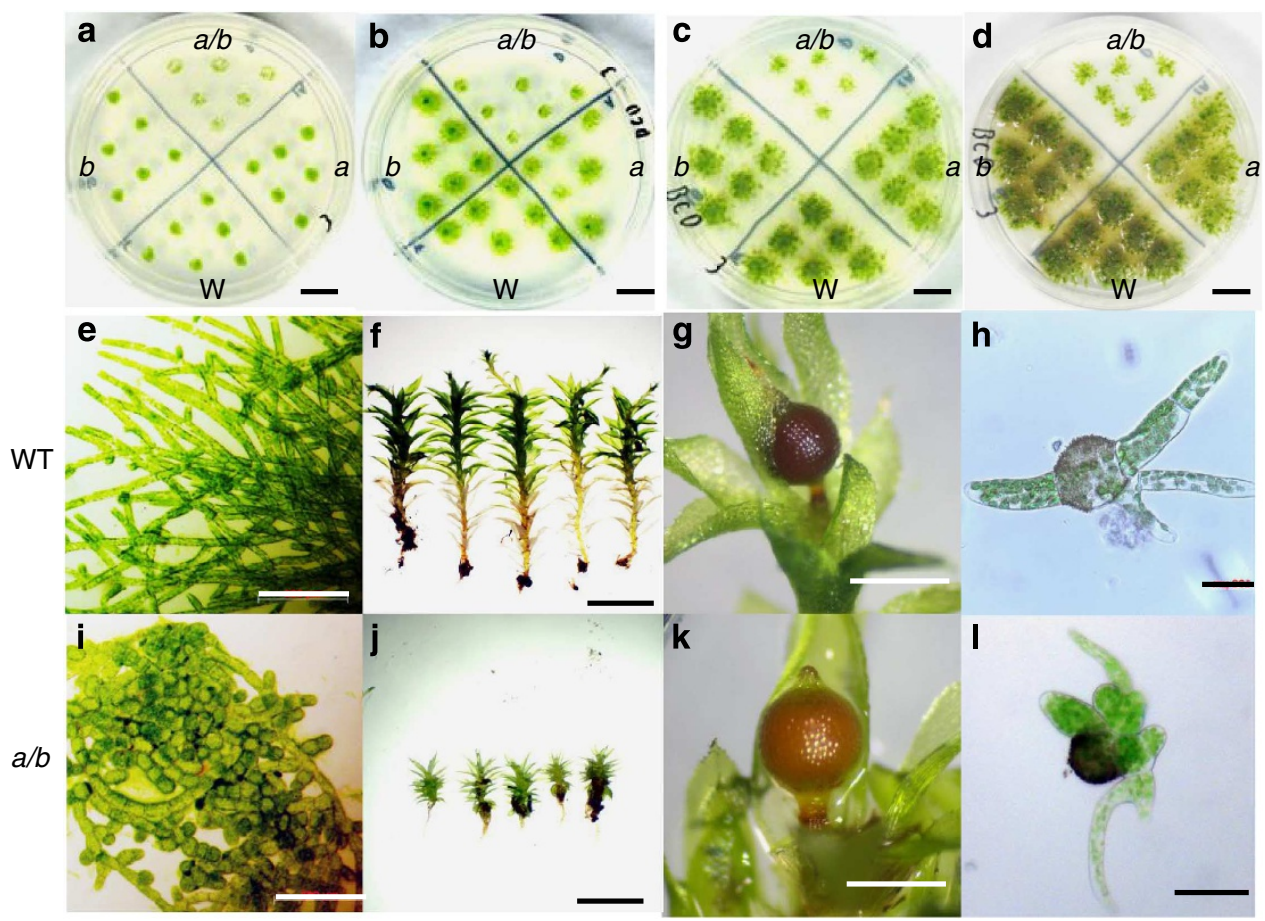

Figure 1 | Growth of ppabi1a/b double disruptants. Protonemal tissue was grown on BCD medium for (a) 1 week, (b) 2 weeks, (c) 4 weeks and (d) 2 months. W, wild type; $a$, ppabila; $b$, ppabilb; $a / b$, ppabila/b. Magnified images of protonemal cells (e,i), gametophores grown on sterile peat pellets $(\mathbf{f}, \mathbf{j})$, sporophytes $(\mathbf{g}, \mathbf{k})$ and germinating spores $(\mathbf{h}, \mathbf{I})$. WT (e-h) and ppabila/b (i-I). Scale bars in $\mathbf{a}-\mathbf{d}, 1 \mathrm{~cm} ; \mathbf{e}, \mathbf{i}, 200 \mu \mathrm{m} ; \mathbf{f}, \mathbf{j}, 5 \mathrm{~mm} ; \mathbf{g}, \mathbf{k}, 0.5 \mathrm{~mm}$; and $\mathbf{h}, \mathbf{l}, 100 \mu \mathrm{m}$.

water-stressed plant body, and are suggested to be involved in protection of cells from desiccation ${ }^{27}$. Because of this highly hydrophilic feature, LEA proteins are often boiling soluble ${ }^{28,29}$. When proteins from a protonemal crude extract were analysed, electrophoretic profiles were similar between WT and transgenic plants (Supplementary Fig. S4); however, the accumulation profiles of boiling-soluble proteins were quite different (Fig. 3a). The ppabila/b plants accumulated boiling-soluble proteins in the absence of exogenously applied $\mathrm{ABA}$, whereas accumulation of boiling-soluble proteins of WT or both single disruptants depended on ABA application. Exogenously applied ABA failed to enhance accumulation of boiling-soluble proteins in the ppabila/b plants. We further performed immunoblot experiments using specific antibodies against dehydrin (Group 2 LEA) or the LEA-like protein 17B9 (protein ID 171483), confirming the specific accumulation of LEA proteins in the ppabila/b plants but not in WT without ABA treatment (Supplementary Fig. S5). We next performed analysis of the soluble sugar accumulation, because levels of soluble sugars associate with ABA-induced freezing tolerance in $P$. patens ${ }^{30}$. The ppabila/b plants accumulated considerable amounts of soluble sugars regardless of ABA application, whereas WT and both single disruptants increased accumulation of soluble sugars in response to ABA application (Fig. 3b). The analysis of soluble sugars showed constitutive accumulation of disaccharide as the major sugar and also detected trisaccharide with the same profile as that of ABAtreated WT (Supplementary Fig. S6). These disaccharides and trisaccharides showing induced accumulation by $\mathrm{ABA}$ in WT were identified as sucrose and theanderose, respectively ${ }^{30}$. We also observed slight accumulation of additional sugar species in the ppabila/b plants, although their molecular characterizations are yet to be performed. These data suggest that the ppabila/b plants constitutively accumulate LEA proteins and soluble sugars at the maximum level even under standard growth conditions, which might be associated with extremely enhanced stress -tolerance.

Role of Group A PP2C in global gene expression. One open question is how globally Group A PP2C regulates ABA-responsive genes in land plants. To investigate the disruption effect of Group A PP2C on global gene expression, we performed microarray analysis of WT and transgenic plants using a custom Physcomitrella oligonucleotide microarray, which carries probes for 33,942 gene models of the Physcomitrella genome, version 1.1 on an Agilent platform. First, we compared expression profiles in the protonemata of the mutants with those of the WT in the absence of exogenous ABA (Fig. 4a and Supplementary Data 1). The results showed that 354 genes, 131 genes and 873 genes were significantly upregulated ( $>2$ fold) in ppabila, ppabilb and ppabila/b, respectively. Approximately $95 \%$ and $62 \%$ of upregulated genes in ppabila and ppabilb, respectively, were also upregulated in ppabila/b. Thus, PpABI1A and PpABI1B appear to be highly redundant, and PpABI1A has a more important role than PpABI1B in the ABA response of protonemata.

To investigate how many ABA-inducible genes are included in the 873 Group A PP2C-regulated genes, we selected 731 genes that were significantly induced ( $>2$-fold) after $3 \mathrm{~h}$ of $1 \mu \mathrm{M} \mathrm{ABA}$ treatment in WT triplicate experiments. The number of ABAinducible genes that were upregulated in the ppabila plants (296 genes) was much greater than in the ppabilb plants (50 genes). Of note, the number of upregulated genes in the ppabila/b plants included 474 of the ABA-inducible genes. These results indicate that Group A PP2C regulates at least $65 \%$ of the ABA-inducible genes. Figure $4 \mathrm{~b}$ shows a cluster analysis of the 911 ABAresponsive genes (731 inducible genes and 180 repressive genes in response to $1 \mu \mathrm{M} \mathrm{ABA}$ for $3 \mathrm{~h}$ ) from the microarray data for WT plants and for the three ppabil transgenics. In the absence of 


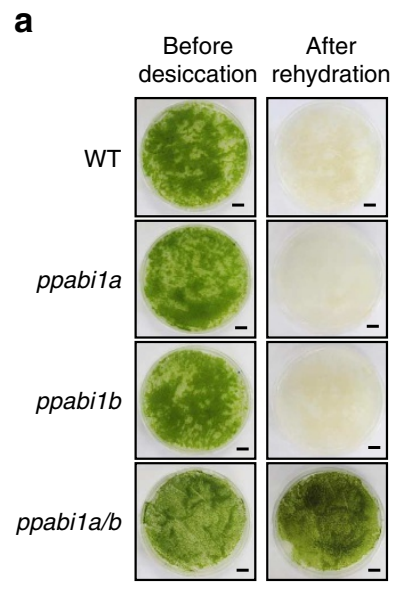

b

b
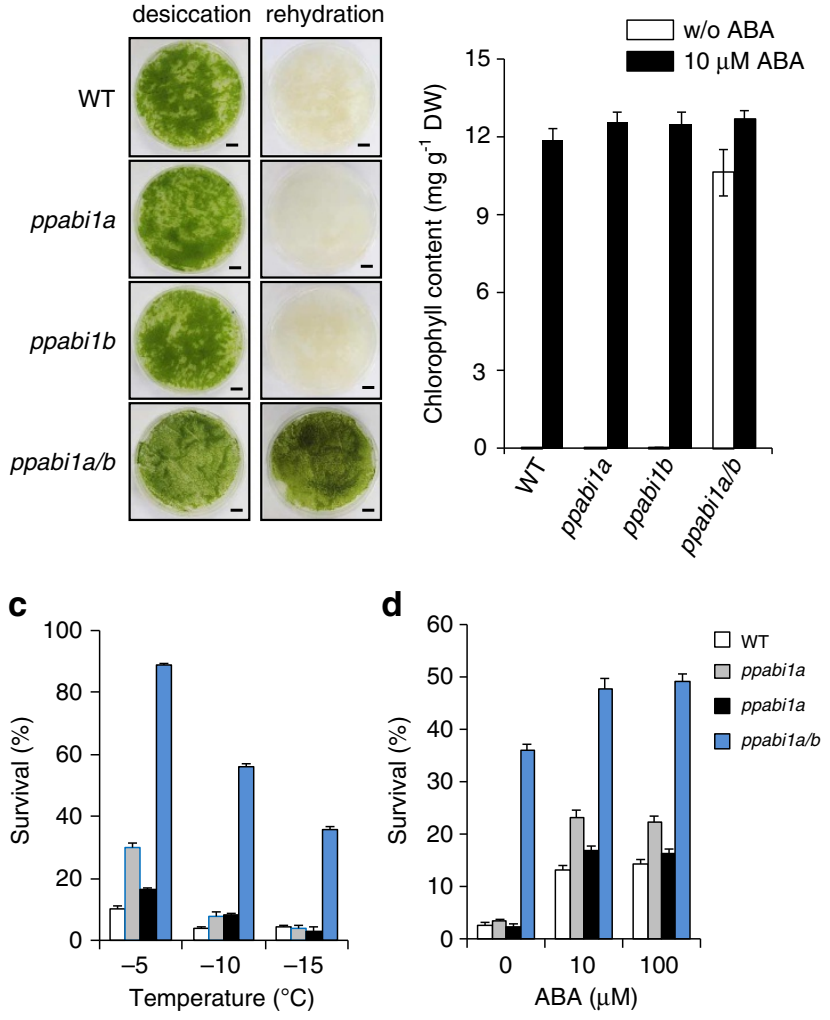

Figure 2 | Disruption of PpABI1 enhanced tolerance to desiccation and freezing in a gene dose-dependent manner. (a) One-week-old protonemata of WT and disruptants (ppabila, ppabilb and ppabila/b) were dried for $24 \mathrm{~h}$, rehydrated with sterile distilled water and incubated for 2 weeks. Scale bars, $1 \mathrm{~cm}$. (b) Chlorophyll content of WT and disruptants (ppabila, ppabilb and ppabila/b), treated with or without ABA $(10 \mu \mathrm{M})$ for $24 \mathrm{~h}$, was determined after desiccation assay. The values are mean \pm s.e. $(n=3)$. (c) One-week-old protonemata of WT and disruptants (ppabila, ppabiib and ppabila/b) were frozen to $-5^{\circ} \mathrm{C},-10^{\circ} \mathrm{C}$ and $-15^{\circ} \mathrm{C}$. Survival was determined by measuring the electrolyte leakage. (d) Protonemata cells were treated with indicated concentrations of $A B A$ for $24 \mathrm{~h}$ and frozen to $-15^{\circ} \mathrm{C}$. The values are mean \pm s.e. $(n=3)$. DW, dry weight.

exogenous $\mathrm{ABA}$, the ppabila/b plants showed the closest expression profile to that of ABA-treated WT. We also compared the expression patterns of the 911 ABA-regulated genes after ABA treatment (Fig. 4c). Compared with the WT, the response of these genes in the ppabila/b plants to exogenous $\mathrm{ABA}$ was greatly reduced, indicating that expression of ABA-regulated genes in the ppabila/b plants is mostly saturated without ABA application. We also confirmed the expression profile of several ABAinducible genes by RNA blot analysis (Fig. $4 \mathrm{~d}$ and Supplementary Fig. S7). Consistent with the results of the microarray analysis, the ppabila/b plants accumulated the transcripts of these $\mathrm{ABA}$-inducible genes in the absence of exogenous $\mathrm{ABA}$, and exogenously applied ABA affected the expression levels only slightly, although some genes still showed significant transient activation by ABA.

We found that considerable numbers of $\mathrm{ABA}$-inducible genes ( $>10$-fold) were under the regulation of Group A PP2C (Supplementary Fig. S8). To understand the impact of the Group A PP2C-mediated gene regulation system on genome-wide ABA response, we traced the time course of whole genes in WT that gave sufficient signal intensity for investigation (14,721 genes)
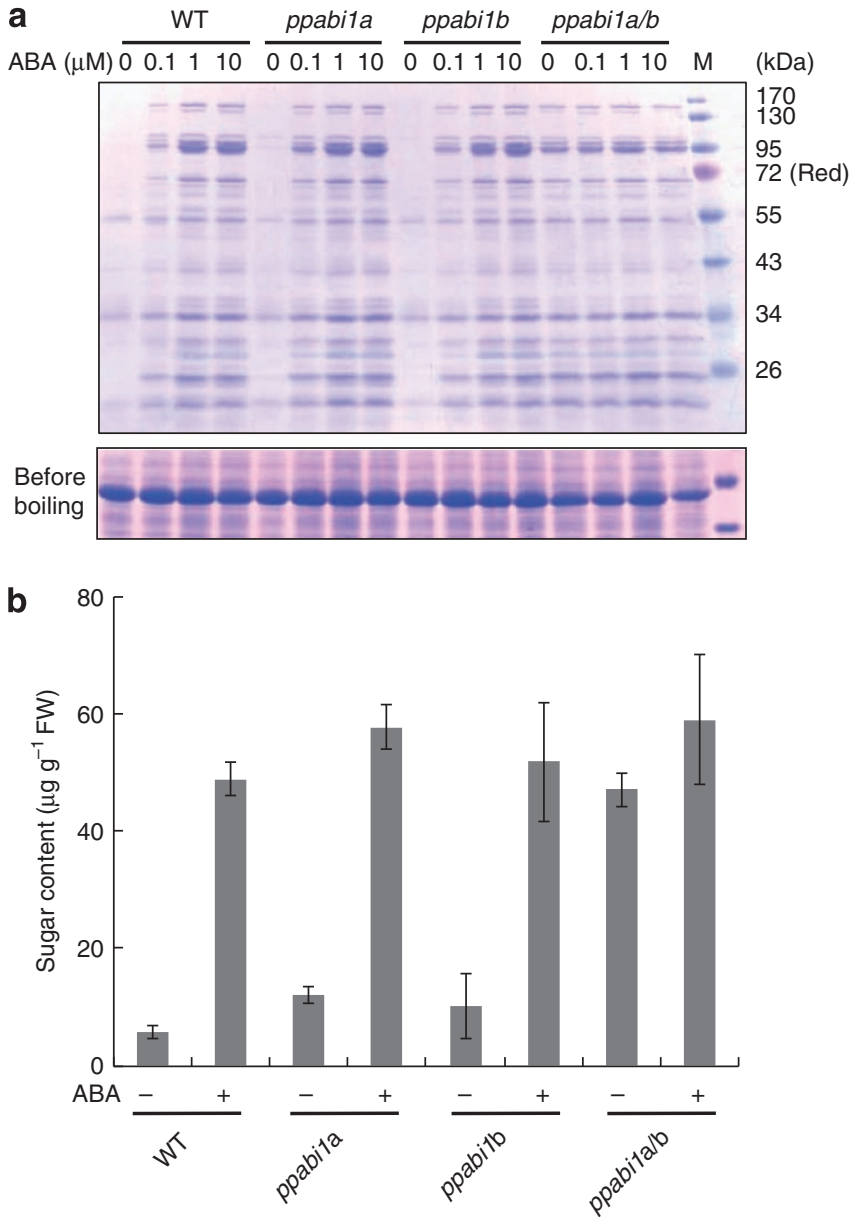

Figure 3 | The ppabi1a/b plants constitutively accumulate boiling-soluble proteins and soluble sugars. (a) Electrophoretic profiles of boiling-soluble proteins prepared from 1-week-old protonemata of WT and disruptants (ppabila, ppabilb and ppabila/b) treated with or without ABA for $24 \mathrm{~h}$. Coomassie Brilliant Blue staining shows an equal amount of proteins subjected to boiling. $M$, molecular weight marker. (b) Soluble sugars extracted from WT and disruptants (ppabila, ppabilb and ppabila/b) treated with or without $A B A(1 \mu M)$ for $24 \mathrm{~h}$ were analysed by anthrone-sulphuric acid assay. The values are mean \pm s.e. $(n=3)$. FW, fresh weight.

and also the time course of the 1,771 Group A PP2C-regulated genes (873 upregulated genes and 898 downregulated genes) (Fig. 5a,b). When the expression profiles of Group A PP2Cregulated genes were removed from the whole gene expression profile (Fig. 5c), genes that were highly induced by $A B A$ had almost disappeared. These results indicate that Group A PP2C is essential for the genome-wide induction system of gene expression in response to ABA. To investigate the main target of Group A PP2C in P. patens, we selected the top 100 genes that showed a high level of induction in ppabila/b plants in the absence of ABA. Although most of the genes were categorized as 'unknown function', it should be noted that 13 LEA genes were included (Supplementary Data 2). To determine how many LEA genes are under the regulation of Group A PP2C, we selected putative LEA genes from $P$. patens genome databases and investigated the ABA responses (Supplementary Data 3). As a result, 77 genes were considered to encode LEA proteins. Among these genes, 67 were included in our custom array chip. A total of 42 of 67 putative LEA genes were upregulated ( $>2$-fold) by exogenous ABA in $\mathrm{WT}$; the remaining genes were not influenced or downregulated by exogenous ABA in WT. In the absence of 
a

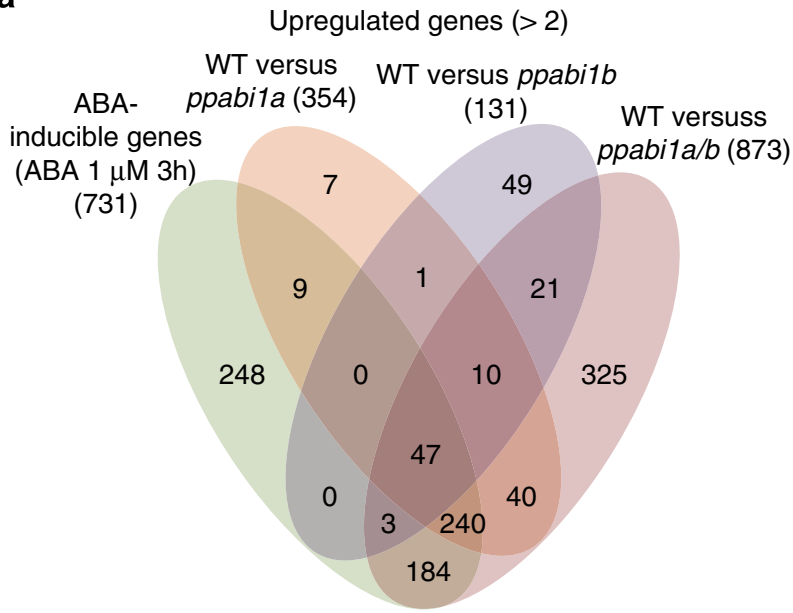

b

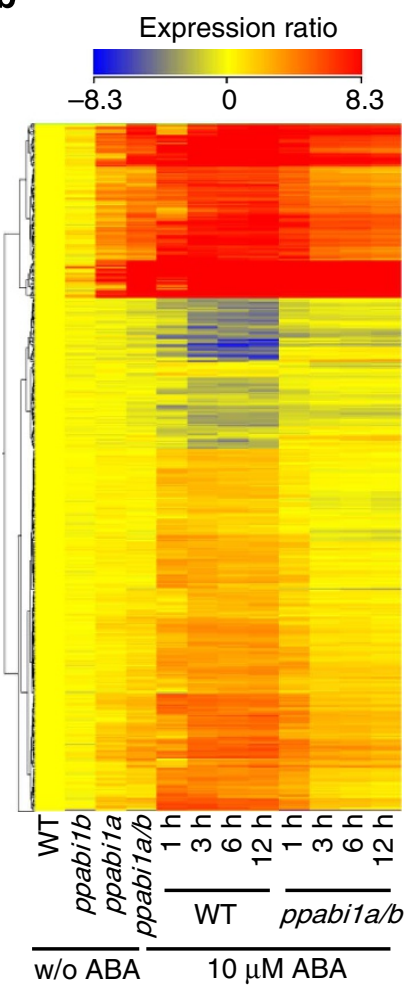

C

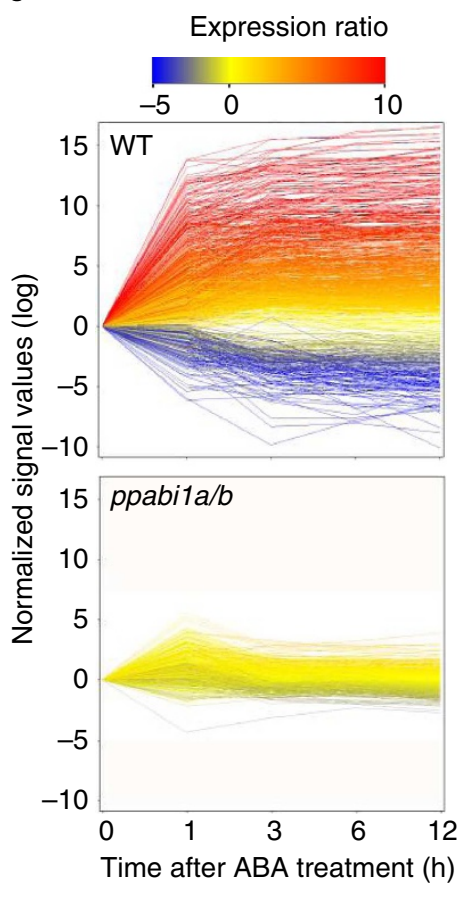

Downregulated genes $(<0.5)$

WT versus ppabila WT versus

(77)

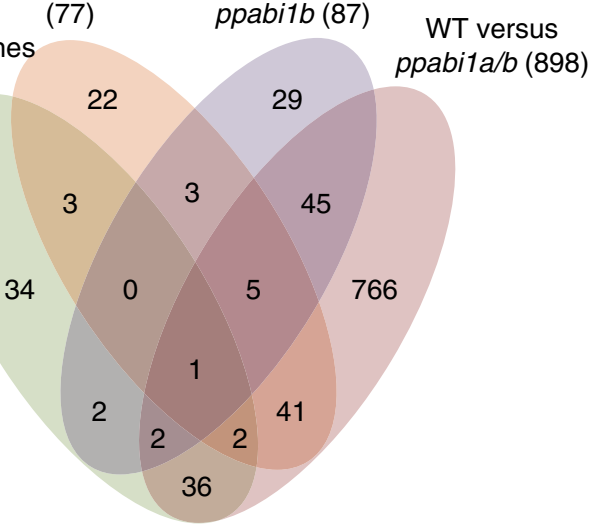

d

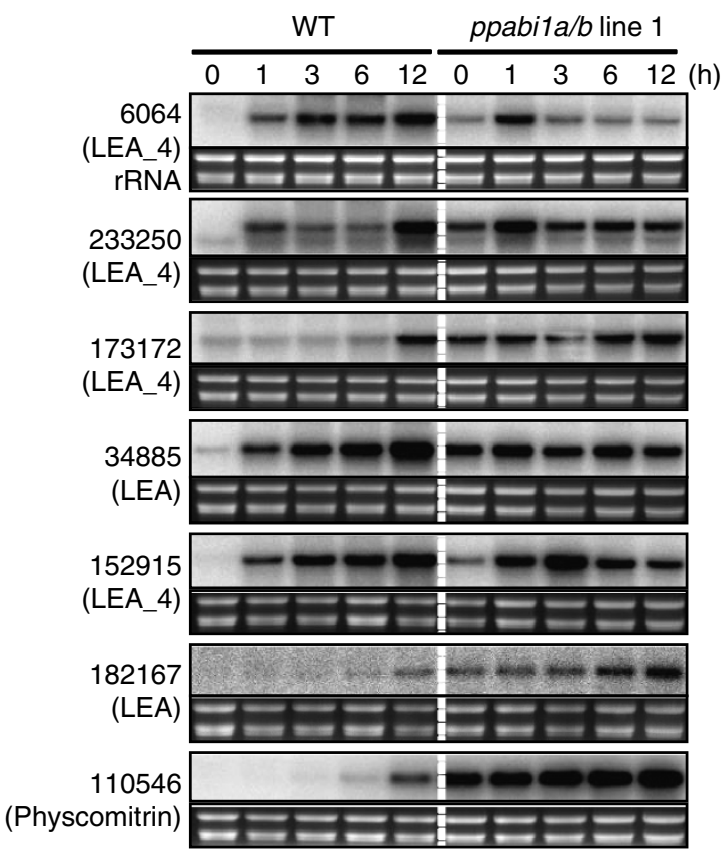

Figure 4 | Comparison of expression profiles of ABA-responsive genes between wild type and ppabi1 disruptants. (a) Venn diagrams show the number of upregulated genes (left) and downregulated genes (right) in ppabil disruptants compared with WT. (b) Hierarchical clustering of 911 ABA-responsive genes. Each gene is represented by a single row of coloured boxes. The colour represents the expression level of the gene. The range of induction and repression is shown from yellow to red and blue. (c) Comparison of expression patterns between ABA-responsive genes in WT and ppabi1 disruptants in time-dependent ABA treatment conditions. Each gene is represented by a coloured line. The colour represents the expression ratio of the gene at $12 \mathrm{~h}$. (d) RNA analysis of ABA-inducible genes in the ppabila/b plant. Protonemata were treated with $10 \mu \mathrm{M} \mathrm{ABA}$ for $0,1,3,6$ and $12 \mathrm{~h}$, and isolated total RNA was subjected to RNA blot analysis for the indicated genes. Genes are indicated by Phypa IDs. For the full blots, see Supplementary Fig. S7.

exogenous $\mathrm{ABA}$, the ppabila/b plants constitutively upregulated 39 out of 42 'ABA upregulated' LEA genes, including dehydrin genes and $17 B 9$, the gene products of which were also detected by immunoblot analysis (Supplementary Fig. S5). These data suggest that expression of LEA genes is strongly associated with Group A PP2C-mediated ABA signalling and with tolerances to desiccation, freezing and salinity in the moss.

Activity of ABA-activated protein kinases in ppabil plants. $\mathrm{ABA}$ induces activity of specific protein kinases in angiosperms. In
Arabidopsis, ABA-activated kinase activity is lost in a triple disruptant of subclass III SnRK2s, suggesting that subclass III SnRK2s are the major kinases for ABA-induced phosphorylation ${ }^{16-19}$. According to the current model of initiation of ABA signalling in which Group A PP2C negatively regulates activity of SnRK2, ppabila/b plants were expected to show constitutive activation of ABA-activated kinase regardless of ABA application. We compared the activity of ABA-activated kinases between WT and the three ppabil transgenics using an in-gel phosphorylation assay with histone as a substrate (Fig. 6). This method has been successfully employed to detect SnRK2 activity 
a

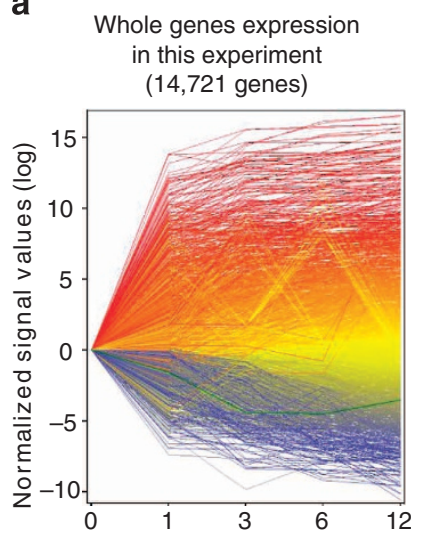

b

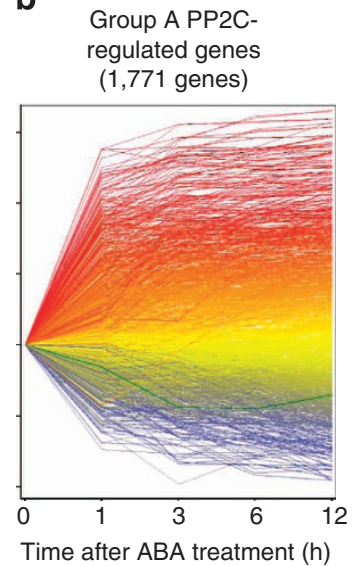

C
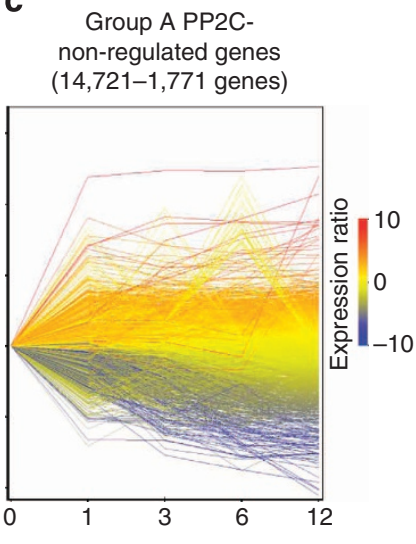

Figure 5 | Comparison of expression patterns between Group A PP2C-regulated and non-regulated genes in ABA treatment conditions. (a) Expression patterns of whole expressed genes (left), (b) Group A PP2C-regulated genes (centre) and (c) Group A PP2C non-regulated genes (right) in ABA-treated wild-type plants are shown as a profile plot. Each gene is represented by a coloured line. The colour represents the expression ratio of the gene at $12 \mathrm{~h}$.

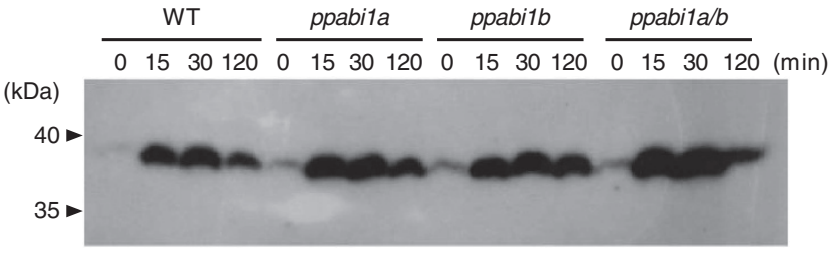

Figure 6 | Effect of PpABI1 disruption on ABA-activated protein kinases. One-week-old protonemata of WT and disruptants (ppabila, ppabilb and ppabila/b) were treated with $10 \mu \mathrm{M} \mathrm{ABA}$ for $0,15,30$ or 120 min and subjected to in-gel phosphorylation assay using histone IIIS as a substrate.

in angiosperms ${ }^{14,31,32}$. As observed in angiosperms, upon ABA application, $P$. patens also rapidly increased kinase activity, detected as one heavy band corresponding to the expected molecular weight (about $40 \mathrm{KDa}$ ) of SnRK2s from $P$. patens, whose genome encodes only subclass III SnRK2 (ref. 33). To our surprise, this ABA-activated kinase activity occurred only slightly in ppabila/b plants in the absence of exogenous $\mathrm{ABA}$ and was markedly activated by ABA application followed by subsequent reduction of the kinase activity. This result indicates that Group A $\mathrm{PP} 2 \mathrm{C}$ is not the major factor regulating ABA-activated kinases and instead acts downstream of the kinases. Our data also suggest the presence of another mechanism for the regulation of kinase activity by ABA.

We investigated the subcellular localization of the green fluorescent protein (GFP)-PpABI1A fusion protein in $P$. patens protonemal cells. The GFP-PpABI1A fusion protein predominantly localized to the nucleus, whereas the Arabidopsis GFPABI1 fusion protein was found in both the cytosol and the nucleus, as has been reported ${ }^{34}$ (Supplementary Fig. S9). These data indicate that Group A PP2C mainly functions in the nucleus in P. patens.

\section{Discussion}

Evolution of the ABA core components PYL/PP2C/SnRK2 in land plants must have had a key role in enabling adaptation to the terrestrial environment. $\mathrm{ABA}$ has been shown to be responsible for stomatal closures and seed desiccation tolerance in angiosperms, both of which are related to adaptation to water-stress conditions. OPEN STOMATA1, a causal gene of an Arabidopsis mutant that has defects in stomatal closure, encodes subclass III
SnRK2.6 (ref. 15). Recently, SnRK2s from the lycophyte Selaginella uncinata and the moss $P$. patens have been shown to rescue the Arabidopsis ost 1 mutant ${ }^{35,36}$, suggesting an evolutionarily conserved function of SnRK2 in the machinery of stomatal closure among land plants. However, what remains a matter of debate is when land plants acquired the ABA-dependent closure system of the stomatal aperture in their evolutionary history ${ }^{35-38}$. Stomata are widely distributed among land plants, except liverworts, indicating that the epidermal apparatus evolved after the separation of liverworts and mosses from a common ancestor more than 400 million years ago. In contrast, ABA signalling that involves Group A PP2C can be found in liverworts ${ }^{39}$, suggesting that the $\mathrm{ABA}$ core components evolved to regulate physiological aspects other than stomata control.

Vegetative desiccation tolerance is considered to have been a key component for successful land colonization in the evolution of green plants ${ }^{40}$, and $\mathrm{ABA}$ has been shown to regulate this physiological response in bryophytes by a mechanism similar to that of seed desiccation tolerance ${ }^{7}$. In this study, we demonstrated that elimination of Group A PP2C is sufficient to confer desiccation tolerance on $P$. patens without any treatment, that is, without exogenous ABA. One might speculate that these phenotypes result from the increased endogenous ABA in the ppabila/b plants. However, we observed a marked activation of ABA-activated kinases after ABA treatment, such as that of the WT. Moreover, we found no significant expression changes in ppabila/b plants of genes encoding 9-cis-epoxycarotenoid dioxygenase or zeaxanthin epoxidase, key enzymes in the ABA biosynthesis pathway ${ }^{41}$. These data indicate that desiccation tolerance of the ppabila/b plants was not the result of the overaccumulation of endogenous ABA. Taken together, the evidence shows that the default status of $P$. patens protonemata is desiccation tolerance, but that Group A PP2C represses this intrinsic feature in the absence of stresses, possibly to prioritize rapid propagation on land. In fact, growth of $P$. patens lacking Group A PP2C was severely retarded. From this point of view, ABA is simply acting to release the intrinsic ability of desiccation tolerance from Group A PP2C regulation.

Elimination of Group A PP2C resulted in activation ( $>2$-fold) of 873 genes as well as repression $(<0.5$-fold $)$ of 898 genes; thus, Group A PP2C regulates expression of at least 1,771 genes. These genes account for about $4.6 \%$ of the total $P$. patens 38,357 gene models (www.cosmoss.org database, Ver. 1.6). Expression profiles of $P$. patens genes that are not regulated by Group A PP2C 
specifically lacked a high level of $A B A$ induction. We also showed that most of the ABA upregulated LEA genes are under the regulation of Group A PP2C in P. patens. These data agree well with the fact that the ppabila/b plants constitutively accumulated boiling-soluble proteins, which is a characteristic feature of LEA proteins. The implication is that expression of LEA genes is strongly associated with Group A PP2C-mediated ABA signalling and with tolerances to desiccation, freezing and salinity in the moss. LEA proteins are widespread in the plant kingdom, from algae to seed plant $\mathrm{s}^{33,42}$. These transcripts accumulate in response to water-stress conditions even in algae. Although ABA can be found in algae, these organisms do not possess core components of ABA signalling, including Group A PP2Cs, and have very low sensitivity to exogenous $A B A^{33}$. Stress-induced expression of LEA genes in algae might be mediated via different mechanisms from land plants. Our findings suggest that the core components of $\mathrm{ABA}$ signalling and regulation of LEA gene expression in response to water-related stresses coevolved in ancestral land plants.

To our surprise, ABA-activated kinases in ppabila/b plants were only slightly activated but not hyperactivated in the absence of $\mathrm{ABA}$, and were considerably activated by $\mathrm{ABA}$ application, followed by rapid reduction. This result raises several issues regarding the proposed role of Group A PP2C. First, Group A PP2Cs may be partly involved but does not have a major role in the regulation in $P$. patens of ABA-activated kinases, probably SnRK2s. Thus, the default status of ABA-activated kinases is 'off, and there is an as-yet-unknown system to activate the kinases. It has been suggested that SnRK2 activity is mainly regulated by reversible phosphorylation/dephosphorylation of a conserved Ser (S175 in SnRK2.6) in the intramolecular domain, called an activation loop $^{43,44}$. In the well-established model in angiosperms, Group A PP2C dephosphorylates the conserved Ser and binds to the kinase domain to block the access of substrates in the absence of $\mathrm{ABA}^{45}$. Upon ABA perception, PYL-related $\mathrm{ABA}$ receptors bind to the catalytic site of Group A PP2 $\mathrm{C}^{22,23,46}$ that inhibits their enzymatic activity. This inhibition in turn allows SnRK2 to become partially active through the structural stabilization of the kinase domain, enabling autophosphorylation of the activation loop ${ }^{47}$. In $P$. patens, removal of Group A PP2C was not sufficient to fully activate the ABAactivated kinases, strongly suggesting PP2C-independent mechanisms for their ABA activation. Phosphorylation of SnRK2 by osmostress and $\mathrm{ABA}$ also involves unidentified staurosporineinsensitive upstream kinases in Arabidopsis ${ }^{43}$. This type of kinase might have a major role in activation of ABA-activated kinases in the basal land plants. We speculate that a strong association between SnRK2 and Group A PP2C might have developed in the vascular plant lineage after separation from the bryophytes. In fact, the ABA box, which is located at the carboxyterminal of Arabidopsis SnRK2 and is responsible for the interaction with Group A PP2Cs ${ }^{45,48}$, is less conserved in the four subclass III SnRK2s encoded in the $P$. patens genome (Supplementary Fig. S10).

The second issue related to the kinase activation results in our ppabila/b plants is that even in the absence of Group A PP2C, negative feedback regulation of $\mathrm{ABA}$-activated kinases was observed. Accumulation of Group A PP2C mRNA is increased after ABA treatment ${ }^{11}$. This response was considered to be the negative feedback regulation of ABA signalling. However, again, our results indicate that Group A PP2C is not a major factor in the negative feedback system for repressing ABA-activated kinases and that there must be another protein phosphatase responsible for their inactivation.

Several reports describe proteins that can interact with Group A PP2C, including SnRK3-type protein kinase SOS2 (ref. 49), a potassium channel AKT3 (ref. 50), ATHB6

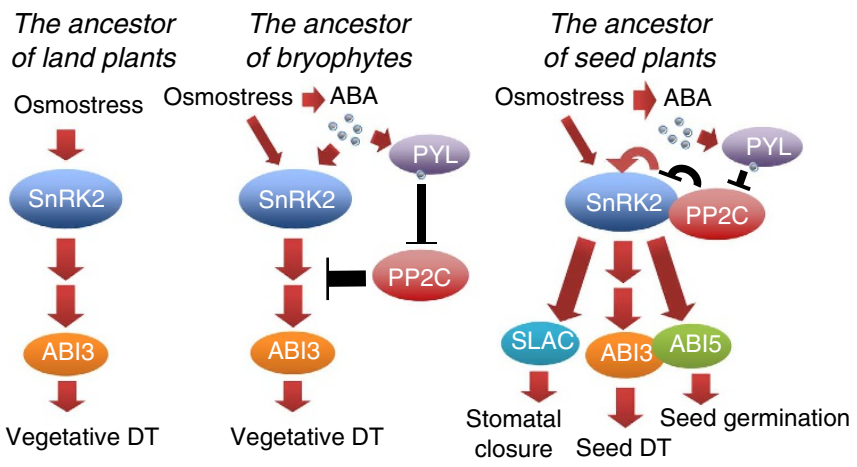

Figure 7 | Evolutionary model of Group A PP2C-mediated ABA

signalling. The ancestor of bryophytes (middle) recruited $\mathrm{PP} 2 \mathrm{C}$ to control the pre-existing pathway for vegetative desiccation tolerance (DT) (left) in an ABA-dependent manner. Note the PP2C action downstream of SnRK2. The ancestor of seed plants (right) developed tight regulation of SnRK2 by PP2C, which enabled integrated regulation of an increased number of signalling pathways downstream of SnRK2.

transcription factor ${ }^{51}$ and SWI3B chromatin remodelling factor $^{52}$. Our data demonstrate that Group A PP2C negatively regulates a signalling pathway downstream of ABA-activated kinases, confirming that Group A PP2C acts at different layers of ABA signalling. As the ppabila/b plants showed constitutive activation of the ABA-signalling pathway that is not necessarily related to the activity of $\mathrm{ABA}$-activated kinases, the target of Group A PP2C must be a key component for acquisition of desiccation tolerance. As intensive nuclear localization of the GFP-PpABI1A fusion protein was observed here in transiently transformed protonemal cells regardless of $\mathrm{ABA}$ application, the major target of Group A PP2C must be present in the nucleus.

We have developed a working model for the evolution of Group A PP2C-mediated ABA signalling in land plants (Fig. 7). Before the emergence of Group A PP2Cs, activation of the pathway for vegetative desiccation tolerance might have been a default status in ancestral land plants, as observed in ppabila/b plants. In the next evolutionary step, the ancestor of bryophytes recruited Group A PP2Cs as key regulators to control this preexisting desiccation tolerance pathway downstream of the kinases in an ABA-dependent manner, with newly evolved $A B A$ receptors, PYLs, for better growth to survive on land. Later during their evolution, the ancestor of seed plants acquired novel SnRK2 substrates, such as ABI5-related bZIPs ${ }^{53,54}$ and SLAC1, a critical anion channel for stomatal closure ${ }^{55,56}$, possibly leading to the emergence of distinct $\mathrm{ABA}$-signalling branches to control seed germination and stomatal closures. The development of a strong interaction between SnRK2 and PP2C would have enabled tight regulation of SnRK2 by PP2C, which might be necessary for control of the increased numbers of downstream signalling pathways of SnRK2.

\section{Methods}

Plant materials. $P$. patens subspecies patens (Gransden) was the WT strain. Protonemal tissue was grown on BCDAT medium at $25^{\circ} \mathrm{C}$ under continuous light $\left(50-80 \mu \mathrm{mol} \mathrm{m}{ }^{-2} \mathrm{~s}^{-1}\right)^{24}$. The gametophores were induced on sterile peat pellets (Jiffy-7, Sakata Seed, Japan) in a plant culture box by culturing at $25^{\circ} \mathrm{C}$ under continuous light for $1-1.5$ months. For induction of sporophytes, gametophore cultures were transferred to $15^{\circ} \mathrm{C}$ under $8 \mathrm{~h}$ light $/ 16 \mathrm{~h}$ dark conditions.

Targeting construct for PpABI1B. To create the $P p A B I 1 B$ targeting construct, the upstream $(3,067 \mathrm{bp})$ and downstream regions $(3,053 \mathrm{bp})$ of the open-reading frame were amplified by PCR using KOD Plus (Toyobo, Japan) with the following primer pairs: $5^{\prime}$-CCGCATTTGCCTAAGACTTC- $3^{\prime} / 5^{\prime}$-GAATTTCCATCCTGCG ACGC- $3^{\prime}$ and $5^{\prime}$-ATAGGTAACCCGTGGATGGT- $3^{\prime} / 5^{\prime}$-AGAGCTACATGCA 
TTTCAAG- $3^{\prime}$ for upstream and downstream regions of $P p A B I 1 B$, respectively. The amplified fragments were verified by sequencing and cloned into both ends of the hygromycin resistance cassette in pBlueScript KS + (Agilent Technologies, USA). Preparation of protoplasts from PpABIIA single-disruptant plants (ppabila) and the transformation were carried out by polyethylene glycol-mediated method ${ }^{24}$ and $25 \mu \mathrm{g} \mathrm{ml}^{-1}$ hygromycin was used for screening of transformants.

Genomic PCR. Genomic DNA was isolated using PhytoPure (GE Healthcare, UK) with an additional RNase treatment. To check homologous recombination at the $5^{\prime}$ - and $3^{\prime}$-flanking regions of the PpABI1B genomic locus, PCR was performed using primer set $A$ for the $5^{\prime}$-recombination and primer set $B$ for the $3^{\prime}$-recombination. The absence of the PpABI1B genomic region was confirmed by PCR using KOD FX (Toyobo, Japan) with primer set C. The PCR condition was 30 cycles at $98^{\circ} \mathrm{C}$ for $10 \mathrm{~s}, 45^{\circ} \mathrm{C}$ for $30 \mathrm{~s}$ and $68^{\circ} \mathrm{C}$ for $15 \mathrm{~s}$. The lack of transcripts corresponding to PpABIIA and/or PpABIIB in the ppabilb and ppabila/b plants was confirmed in microarray analysis (see below). The primer sets are described in Supplementary Table S1.

Southern blot analysis. The genomic DNA of the ppabila/b plants was extracted using PhytoPure (GE Healthcare, UK) with an additional RNase treatment. The probe for hygromycin was amplified using a PCR DIG Probe Synthesis Kit (Roche Applied Science, Germany) with PCR and the following primers: $5^{\prime}$-CCGGAGAT TACAATGGACGA-3' $/ 5^{\prime}$-TTCTTCCACGAAGCTCCTCG-3'. Insertion of the $P$ PABI1B disruption construct was analysed using $2 \mu \mathrm{g}$ of genomic DNA digested with NdeI. Digested DNA was separated on $0.6 \%$ agarose gels and transferred to nylon filters (Hybond-N +, GE Healthcare). The membrane was hybridized with DIG-labelled probes in DIG Easy Hyb buffer (Roche Applied Science) at $41^{\circ} \mathrm{C}$ overnight. After washing, the membrane was treated once with blocking buffer for $1 \mathrm{~h}$ at room temperature. The membrane was reacted with Anti-Dig (Roche Applied Science) for $1 \mathrm{~h}$ at room temperature. Detection was carried out with CDP-Star (Roche Applied Science) for $5 \mathrm{~min}$ at room temperature in the dark. The signal was detected by ChemiDoc XRS Plus (Bio-Rad Laboratories, USA).

RNA blot analysis. Total RNAs were extracted from protonemal tissues using the RNeasy plant mini Kit (QIAGEN, USA). Ten micrograms of total RNA was separated by a formaldehyde-denaturing agarose gel and transferred onto a nylon membrane. Probes were generated by PCR of complementary DNA prepared from ABA-treated WT plants. The primer sets are described in Supplementary Data 4. PCR fragments were purified using the Gel Extraction kit (QIAGEN, USA). Hybridization with ${ }^{32} \mathrm{P}$-labelled DNA probes was carried out ${ }^{24}$. BAStaion 2500 (Fuji Film, Japan) was used for visualization of the blots.

Protein analyses. Protonemal cells were homogenized and extracted with $50 \mathrm{mM}$ Tris (pH 7.5), $100 \mathrm{mM} \mathrm{NaCl}, 0.1 \%$ Triton X-100, 0.1 mM EDTA, 2 mM dithiothreitol (DTT) and $1 \mathrm{mM}$ phenylmethylsulphonyl fluoride on ice. After centrifugation at $14,000 \mathrm{~g}$ for $10 \mathrm{~min}$ at $4{ }^{\circ} \mathrm{C}$, supernatants were used as crude fractions. The proteins in the crude fractions, adjusted to equal concentrations, were boiled for $10 \mathrm{~min}$, and insoluble pellets were removed by centrifugation at $14,000 \mathrm{~g}$ for $10 \mathrm{~min}$ at $4{ }^{\circ} \mathrm{C}$. These soluble fractions were used as boiling-soluble proteins. The boiling-soluble proteins corresponding to $20 \mu \mathrm{g}$ of crude proteins were used for gel electrophoresis. The crude proteins and boiling-soluble proteins were separated on $12 \%(\mathrm{w} / \mathrm{v})$ SDS-polyacrylamide gels. After electrophoresis, proteins were stained using Coomassie Brilliant Blue.

Soluble sugar measurement. Protonema tissues were homogenized at $0^{\circ} \mathrm{C}$ in $5 \mathrm{ml}$ of EtOH- $\mathrm{H}_{2} \mathrm{O}(4: 1)$. The homogenate was centrifuged at $14,000 \mathrm{~g}$ for $5 \mathrm{~min}$ at $4{ }^{\circ} \mathrm{C}$, and the supernatant was recovered. After evaporation of ethanol, the samples were dissolved in distilled $\mathrm{H}_{2} \mathrm{O}(0.5 \mathrm{ml})$ and centrifuged at $14,000 \mathrm{~g}$ for $5 \mathrm{~min}$ at $4{ }^{\circ} \mathrm{C}$ to remove any debris. Soluble sugars in the supernatant were quantified by the anthrone-sulphuric acid assay using glucose as a standard ${ }^{57}$.

Desiccation assay. Protonemal tissues grown for 7 days on cellophane-layered BCDAT medium, treated with or without ABA for $24 \mathrm{~h}$, were transferred to an empty Petri dish and desiccated inside a laminar flow hood for $24 \mathrm{~h}$. The desiccated tissues were rehydrated with $5 \mathrm{ml}$ of sterile water. These rehydrated tissues were then incubated under the standard growth conditions described above for 2 weeks. Measurement of water content was calculated as the difference between the fresh weight and the oven dry weight, divided by the oven dry weight ${ }^{6}$.

Measurement of chlorophyll content. The chlorophyll of the homogenized protonemal tissue was extracted with $10 \mathrm{ml}$ of $80 \%(\mathrm{v} / \mathrm{v})$ acetone. To remove cell debris, the samples were centrifuged for $5 \mathrm{~min}$ at $9,000 \mathrm{~g}$. Supernatants were measured at wavelengths of 645 and $663 \mathrm{~nm}$ for the chlorophyll quantification. After measurement, the supernatant was dried up in the initial tube at room temperature until a stable dry weight of each sample was obtained. The total chlorophyll was calculated as follows: $\mathrm{mg}$ chlorophyll per $\mathrm{g}$ dry weight $=$ $\left[\left(\mathrm{A}_{663}\right)(0.00802)+\left(\mathrm{A}_{645}\right)(0.0202)\right] \times 50 /$ dry weight.
Measurement of freezing tolerance. Freezing tolerance was determined by measurement of electrolyte leakage after equilibrium freezing to various subzero temperatures. The amount of electrolyte leakage from the freeze-thawed tissues (Eft) was then determined by measuring electroconductivity. After the measurement, the tubes containing the samples were boiled for $10 \mathrm{~min}$, and the amount of electrolytes after boiling $(\mathrm{Eb})$ was determined. Survival of the cells was represented as the $\mathrm{Eft} / \mathrm{Eb}$ ratio (\%).

Transcriptome analysis. Microarray analysis was performed using a $P$. patens custom oligonucleotide microarray. Gene sequences used for oligonucleotide probe development were obtained from $P$. patens subsp. patens version 1.1 genome annotation ${ }^{58}$. For 35,938 gene models, 60 -mer oligonucleotide probes were designed from the $3^{\prime}$-region of the sequence in consideration of Tm value, CG content, matching against expressed sequence tag and gene specificity; finally, 33,942 gene-specific probes were mounted in an Agilent format microarray.

Total RNA was extracted from ABA-treated protonemal tissues of WT plants and from the three ppabil transgenics. For definition of significant ABA-regulated genes in WT and identification of differentially expressed genes in the three ppabil transgenics, three biological replicate samples were analysed. The microarray experiments were performed using the Agilent one-colour method according to the manufacturer's instructions. Spot signal values were calculated by Feature Extraction ver. 9.1 software (Agilent Technologies). Digitalized signal values were imported into GeneSpring GX software (Agilent Technologies) and normalized to shift to the 75th percentile. Quality control was carried out with a filter for intensity value; probes with intensity values less than 500 in any experiments were removed. After filtering for intensity value, a total of 14,721 probes were subjected to further analysis. Fold-change analysis was performed on triple replicate samples to determine ABA-regulated genes and PpABI1-regulated genes. A twofold expression cut-off was considered to determine upregulated and downregulated genes (Supplementary Data 1). The false discovery rate for the analysis was 0.05 .

In-gel phosphorylation assay. One-week-old protonemata were treated with $10 \mu \mathrm{M}$ ABA. Tissues were crushed by a mortar in liquid nitrogen and homogenized in extraction buffer containing $50 \mathrm{mM}$ HEPES pH 7.5, $5 \mathrm{mM}$ EDTA, $5 \mathrm{mM}$ EGTA $25 \mathrm{mM} \mathrm{NaF}, 2 \mathrm{mM}$ DTT, $1 \mathrm{mM} \mathrm{Na}_{3} \mathrm{VO}_{4}, 2 \mathrm{mg} \mathrm{ml}^{-1}$ leupeptin, $2 \mathrm{mg} \mathrm{ml}^{-1}$ pepstatin A, $2 \mathrm{mM}$ phenylmethylsulphonyl fluoride, $50 \mathrm{mM} \beta$-glycerophosphate and $20 \%$ glycerol. Equal amounts of total soluble proteins were electrophoresed in $10 \%$ SDS-polyacrylamide gel to which $0.5 \%$ (w/v) histone IIIS (Sigma, MO, USA) had been added. After denaturation and renaturation procedures, the kinase activity was detected by incubation with $50 \mu \mathrm{M} \gamma \gamma_{-}{ }^{32} \mathrm{P}$-labelled ATP in a buffer containing $40 \mathrm{mM}$ Hepes- $\mathrm{KOH}$ (pH 7.5), $5 \mathrm{mM} \mathrm{MgCl}_{2}, 2 \mathrm{mM}$ DTT and $0.1 \mathrm{mM}$ EGTA, followed by washing and autoradiography ${ }^{5}$.

\section{References}

1. Finkelstein, R. R., Gampala, S. S. L. \& Rock, C. D. Abscisic acid signaling in seeds and seedlings. Plant Cell 14(Suppl): S15-S45 (2002).

2. Finkelstein, R., Reeves, W., Ariizumi, T. \& Steber, C. Molecular Aspects of Seed Dormancy*. Annu. Rev. Plant Biol. 59, 387-415 (2008).

3. Oliver, M. J., Velten, J. \& Mishler, B. D. Desiccation tolerance in bryophytes: a reflection of the primitive strategy for plant survival in dehydrating habitats? Integr. Comp. Biol. 45, 788-799 (2005).

4. Bopp, M. \& Werner, O. Abscisic acid and desiccation tolerance in mosses. Botanica Acta 106, 103-106 (1993).

5. Werner, O., Ros Espín, R. M., Bopp, M. \& Atzorn, R. Abscisic-acid-induced drought tolerance in Funaria hygrometrica Hedw. Planta 186, 99 (1991).

6. Koster, K., Balsamo, R., Espinoza, C. \& Oliver, M. Desiccation sensitivity and tolerance in the moss Physcomitrella patens: assessing limits and damage. Plant Growth Regul. 62, 293-302 (2010).

7. Khandelwal, A. et al. Role of ABA and ABI3 in desiccation tolerance. Science 327, 546 (2010).

8. Meyer, K., Leube, M. P. \& Grill, E. A protein phosphatase 2 C involved in ABA signal transduction in Arabidopsis thaliana. Science 264, 1452-1455 (1994).

9. Leung, J. et al. Arabidopsis ABA response gene ABI1: features of a calciummodulated protein phosphatase. Science 264, 1448-1452 (1994).

10. Leung, J., Merlot, S. \& Giraudat, J. The Arabidopsis abscisic acid-insensitive2 (ABI2) and ABI1 genes encode homologous protein phosphatases $2 \mathrm{C}$ involved in abscisic acid signal transduction. Plant Cell 9, 759-771 (1997).

11. Saez, A. et al. Gain-of-function and loss-of-function phenotypes of the protein phosphatase 2C HAB1 reveal its role as a negative regulator of abscisic acid signalling. Plant J. 37, 354-369 (2004).

12. Nishimura, N. et al. ABA-Hypersensitive Germination1 encodes a protein phosphatase $2 \mathrm{C}$, an essential component of abscisic acid signaling in Arabidopsis seed. Plant J. 50, 935-949 (2007).

13. Cutler, S. R., Rodriguez, P. L., Finkelstein, R. R. \& Abrams, S. R. Abscisic acid: emergence of a core signaling network. Annu. Rev. Plant Biol. 61, 651-679 (2010).

14. Yoshida, R. et al. ABA-activated SnRK2 protein kinase is required for dehydration stress signaling in Arabidopsis. Plant Cell Physiol. 43, 1473-1483 (2002). 
15. Mustilli, A.-C., Merlot, S., Vavasseur, A., Fenzi, F. \& Giraudat, J. Arabidopsis OST1 protein kinase mediates the regulation of stomatal aperture by abscisic acid and acts upstream of reactive oxygen species production. Plant Cell 14, 3089-3099 (2002)

16. Fujita, Y. et al. Three SnRK2 protein kinases are the main positive regulators of abscisic acid signaling in response to water stress in Arabidopsis. Plant Cell Physiol. 50, 2123-2132 (2009).

17. Nakashima, K. et al. Three Arabidopsis SnRK2 protein kinases, SRK2D/ SnRK2.2, SRK2E/SnRK2.6/OST1 and SRK2I/SnRK2.3, involved in ABA signaling are essential for the control of seed development and dormancy. Plant Cell Physiol. 50, 1345-1363 (2009).

18. Fujii, H. \& Zhu, J.-K. Arabidopsis mutant deficient in 3 abscisic acid-activated protein kinases reveals critical roles in growth, reproduction, and stress. Proc. Natl Acad. Sci. USA 106, 8380-8385 (2009).

19. Umezawa, T. et al. Type $2 \mathrm{C}$ protein phosphatases directly regulate abscisic acid-activated protein kinases in Arabidopsis. Proc. Natl Acad. Sci. USA 106, 17588-17593 (2009)

20. Ma, Y. et al. Regulators of PP2C phosphatase activity function as abscisic acid sensors. Science 324, 1064-1068 (2009).

21. Park, S.-Y. et al. Abscisic acid inhibits type $2 \mathrm{C}$ protein phosphatases via the PYR/PYL family of START proteins. Science 324, 1068-1071 (2009).

22. Miyazono, K.-I. et al. Structural basis of abscisic acid signalling. Nature 462 , 609-614 (2009).

23. Melcher, K. et al. A gate-latch-lock mechanism for hormone signalling by abscisic acid receptors. Nature 462, 602-608 (2009).

24. Komatsu, K. et al. Functional analyses of the ABI1-related protein phosphatase type $2 \mathrm{C}$ reveal evolutionarily conserved regulation of abscisic acid signaling between Arabidopsis and the moss Physcomitrella patens. Plant Mol. Biol. 70, 327-340 (2009).

25. Goode, J. A., Stead, A. D. \& Duckett, J. G. Redifferentiation of moss protonemata: an experimental and immunofluorescence study of brood cell formation. Can. J. Bot. 71, 1510-1519 (1993).

26. Christianson, M. L. ABA prevents the second cytokinin-mediated event during the induction of shoot buds in the moss Funaria hygrometrica. Am. J. Bot. 87, 1540-1545 (2000).

27. Shih, M. D., Hoekstra, F. A. \& Hsing, Y. I. C. Late embryogenesis abundant proteins. Advances in Botanical Research. Chapter 4 48, 211-255 (2008).

28. Knight, C. D. et al. Molecular responses to abscisic acid and stress are conserved between moss and cereals. Plant Cell 7, 499-506 (1995).

29. Minami, A. et al. Cold acclimation in bryophytes: low-temperature-induced freezing tolerance in Physcomitrella patens is associated with increases in expression levels of stress-related genes but not with increase in level of endogenous abscisic acid. Planta 220, 414-423 (2005).

30. Nagao, M. et al. Accumulation of theanderose in association with development of freezing tolerance in the moss Physcomitrella patens. Phytochemistry 67, 702-709 (2006).

31. Chae, M. J. et al. A rice dehydration-inducible SNF1-related protein kinase 2 phosphorylates an abscisic acid responsive element-binding factor and associates with ABA signaling. Plant Mol. Biol. 63, 151-169 (2007).

32. Li, J. \& Assmann, S. M. An abscisic acid-activated and calcium-independent protein kinase from guard cells of fava bean. Plant Cell 8, 2359-2368 (1996)

33. Takezawa, D., Komatsu, K. \& Sakata, Y. ABA in bryophytes: how a universal growth regulator in life became a plant hormone? J. Plant Res. 124, 437-453 (2011).

34. Moes, D., Himmelbach, A., Korte, A., Haberer, G. \& Grill, E. Nuclear localization of the mutant protein phosphatase abil is required for insensitivity towards ABA responses in Arabidopsis. Plant J. 54, 806-819 (2008).

35. Chater, C. et al. Regulatory mechanism controlling stomatal behavior conserved across 400 million years of land plant evolution. Curr. Biol. 21, 1025-1029 (2011).

36. Ruszala, E. M. et al. Land plants acquired active stomatal control early in their evolutionary history. Curr. Biol. 21, 1030-1035 (2011).

37. Brodribb, T. J. \& McAdam, S. A. M. Passive origins of stomatal control in vascular plants. Science 331, 582-585 (2011)

38. McAdam, S. A. M. \& Brodribb, T. J. Fern and lycophyte guard cells do not respond to endogenous abscisic acid. Plant Cell 24, 1510-1521 (2012).

39. Tougane, K. et al. Evolutionarily conserved regulatory mechanisms of abscisic acid signaling in land plants: characterization of abscisic acid insensitivel-like type 2C protein phosphatase in the liverwort Marchantia polymorpha. Plant Physiol. 152, 1529-1543 (2010).

40. Oliver, M. J., Tuba, Z. \& Mishler, B. D. The evolution of vegetative desiccation tolerance in land plants. Plant Ecol. 151, 85-100 (2000).

41. Nambara, E. \& Marion-Poll, A. Abscisic acid biosynthesis and catabolism. Annu. Rev. Plant Biol. 56, 165-185 (2005).

42. Battaglia, M., Olvera-Carrillo, Y., Garciarrubio, A., Campos, F. \& Covarrubias, A. A. The enigmatic LEA proteins and other hydrophilins. Plant Physiol. 148, 6-24 (2008).

43. Boudsocq, M., Droillard, M.-J., Barbier-Brygoo, H. \& Laurière, C. Different phosphorylation mechanisms are involved in the activation of sucrose non-fermenting 1 related protein kinases 2 by osmotic stresses and abscisic acid. Plant Mol. Biol. 63, 491-503 (2007).

44. Belin, C. et al. Identification of features regulating OST1 kinase activity and OST1 function in guard cells. Plant Physiol. 141, 1316-1327 (2006).

45. Soon, F.-F. et al. Molecular mimicry regulates ABA signaling by SnRK2 kinases and PP2C phosphatases. Science 335, 85-88 (2012).

46. Nishimura, N. et al. Structural mechanism of abscisic acid binding and signaling by dimeric PYR1. Science 326, 1373-1379 (2009).

47. Ng, L.-M. et al. Structural basis for basal activity and autoactivation of abscisic acid (ABA) signaling SnRK2 kinases. Proc. Natl Acad. Sci. USA 108, 21259-21264 (2011).

48. Yoshida, R. et al. The regulatory domain of SRK2E/OST1/SnRK2.6 interacts with ABI1 and integrates abscisic acid (ABA) and osmotic stress signals controlling stomatal closure in Arabidopsis. J. Biol. Chem. 281, 5310-5318 (2006).

49. Ohta, M., Guo, Y., Halfter, U. \& Zhu, J.-K. A novel domain in the protein kinase SOS2 mediates interaction with the protein phosphatase 2C ABI2. Proc. Natl Acad. Sci. USA 100, 11771-11776 (2003).

50. Vranová, E. et al. The AKT3 potassium channel protein interacts with the AtPP2CA protein phosphatase 2C. J. Exp. Bot. 52, 181-182 (2001)

51. Himmelbach, A., Hoffmann, T., Leube, M., Höhener, B. \& Grill, E. Homeodomain protein ATHB6 is a target of the protein phosphatase ABI1 and regulates hormone responses in Arabidopsis. EMBO J. 21, 3029-3038 (2002).

52. Saez, A., Rodrigues, A., Santiago, J., Rubio, S. \& Rodriguez, P. L. HAB1-SWI3B interaction reveals a link between abscisic acid signaling and putative SWI/SNF chromatin-remodeling complexes in Arabidopsis. Plant Cell 20, 2972-2988 (2008).

53. Furihata, T. et al. Abscisic acid-dependent multisite phosphorylation regulates the activity of a transcription activator AREB1. Proc. Natl Acad. Sci. USA 103 1988-1993 (2006).

54. Kobayashi, Y. et al. Abscisic acid-activated SNRK2 protein kinases function in the gene-regulation pathway of ABA signal transduction by phosphorylating ABA response element-binding factors. Plant J. 44, 939-949 (2005).

55. Geiger, D. et al. Activity of guard cell anion channel SLAC1 is controlled by drought-stress signaling kinase-phosphatase pair. Proc. Natl Acad. Sci. USA 106, 21425-21430 (2009).

56. Lee, S. C., Lan, W., Buchanan, B. B. \& Luan, S. A protein kinase-phosphatase pair interacts with an ion channel to regulate $\mathrm{ABA}$ signaling in plant guard cells. Proc. Natl Acad. Sci. USA 106, 21419-21424 (2009).

57. Yemm, E. W. \& Willis, A. J. The estimation of carbohydrates in plant extracts by anthrone. Biochem J. 57, 508-514 (1954).

58. Rensing, S. A. et al. The Physcomitrella genome reveals evolutionary insights into the conquest of land by plants. Science 319, 64-69 (2008).

59. Bhyan, S. B. et al. Cold acclimation in the moss Physcomitrella patens involves abscisic acid-dependent signaling. J. Plant Physiol. 169, 137-145 (2012).

\section{Acknowledgements}

We thank R. Kaida and T. Takada (Tokyo University of Agriculture, Japan) for technical assistance. We also thank R.S. Quatrano and P.-F. Perroud (Washington University, St. Louis, USA) for their valuable discussion and suggestions. This research was supported by an Advanced Research Project of Tokyo University of Agriculture (to Y.S.)

\section{Author contributions}

T.T., T.H. and Y.S. conceived the project and designed the experiments together with K.K. K.K. and N.S. performed most of the experiments. Y.N., M.N. and H.O. generated all transgenic lines of $P$. patens by gene targeting. D.T. performed the in-gel phosphorylation assay. M.T. and M.S. performed microarray hybridization and scanning. Y.S. wrote the paper together with K.K. and N.S.

\section{Additional information}

Accession codes: The microarray data were deposited to NCBI Gene Expression Omnibus (accession GSE37694).

Supplementary Information accompanies this paper at http://www.nature.com/ naturecommunications

Competing financial interests: The authors declare no competing financial interests

Reprints and permission information is available online at http://npg.nature.com/ reprintsandpermissions/

How to cite this article: Komatsu, K. et al. Group A PP2Cs evolved in land plants as key regulators of intrinsic desiccation tolerance. Nat. Commun. 4:2219 doi: 10.1038/ ncomms3219 (2013)

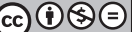

This work is licensed under a Creative Commons AttributionNonCommercial-NoDerivs 3.0 Unported License. To view a copy of this license, visit http://creativecommons.org/licenses/by-nc-nd/3.0/ 